\title{
Elemental analysis of Cosmic-Ray flux with DAMPE
}

\author{
Ya-Peng Zhang ${ }^{* 1,2}$, Ming-Yang Cui ${ }^{3}$, Tie-Kuang Dong ${ }^{3}$, Antonio Surdo ${ }^{4}$, Li-Bo $\mathrm{Wu}^{5,6}$, \\ Yong-Jie Zhang ${ }^{1}$, Cheng-Ming Liu ${ }^{5,6}$, on behalf of the DAMPE collaboration ${ }^{\dagger \ddagger}$ \\ ${ }^{1}$ Institute of Modern Physics, Chinese Academy of Sciences, Lanzhou 730000, China \\ ${ }^{2}$ University of Chinese Academy of Sciences, Beijing 100049, China \\ ${ }^{3}$ Key Laboratory of Dark Matter and Space Astronomy, Purple Mountain Observatory, Chinese \\ Academy of Sciences, Nanjing 210034, China \\ ${ }^{4}$ Istituto Nazionale di Fisica Nucleare (INFN) - Sezione di Lecce, I-73100, Lecce, Italy \\ 5 State Key Laboratory of Particle Detection and Electronics, University of Science and \\ Technology of China,Hefei, Anhui, China, 230026 \\ ${ }^{6}$ Department of Modern Physics, University of Science and Technology of China, Hefei, Anhui, \\ China, 230026 \\ E-mail: y.p.zhang@impcas.ac.cn
}

\begin{abstract}
Dark Matter Particle Explorer (DAMPE) is a satellite-borne detector devoted to measure the fluxes of high-energy cosmic rays (electrons and positrons, photons, protons and nuclei). The DAMPE covers energies up to $10 \mathrm{TeV}$ for electrons and photons and up to $100 \mathrm{TeV}$ for charged nuclei. DAMPE has been launched on December 17th, 2015, and it runs smoothly since then. The Plastic Scintillator Detector (PSD) is designed to accurately measure the charge of cosmic-ray particle and as a veto for Gamma-ray detection. By means of the PSD performance, DAMPE is capable of studying the elemental composition of charged cosmic rays up to Nickel $(Z=28)$. In this contribution, the PSD performances and the status of cosmic-nuclei analysis, with preliminary results on carbon analysis, ultra-heavy nuclei and fractional charge particle searching with DAMPE are presented.
\end{abstract}

36th International Cosmic Ray Conference -ICRC2019-

July 24th - August 1st, 2019

Madison, WI, U.S.A.

\footnotetext{
* Speaker.

${ }^{\dagger}$ For collaboration list see PoS(ICRC2019)1177

¥The DAMPE mission is funded by the strategic priority science and technology projects in space science of Chinese Academy of Sciences. In China the data analysis was supported in part by the National Key Research and Development Program of China (No. 2016YFA0400200), the National Natural Science Foundation of China (No. 11525313, 11622327, 11722328, 11673047, 11205206, 11703062,U1738205, U1738207, U1738208, U1738127), the strategic priority science and technology projects of Chinese Academy of Sciences (No. XDA15051100), the 100 Talents Program of Chinese Academy of Sciences, and the Young Elite Scientists Sponsorship Program. In Europe the activities and the data analysis are supported by the Swiss National Science Foundation (SNSF), Switzerland; the National Institute for Nuclear Physics (INFN), Italy.
} 


\section{Introduction}

Measuring fluxes of cosmic-ray nuclei as a function of energy is important for studying cosmicray origin, acceleration mechanism and their propagation in the interstellar medium $[1,2]$. The DArk Matter Particle Explorer (DAMPE) [3] is a satellite-borne spectrometer for detection of cosmic-rays (electron, gamma-ray, proton and nuclei). DAMPE has been launched on December 17th, 2015 and operates on a sun-synchronous orbit at the altitude of $500 \mathrm{~km}$ with an inclination angle of $97^{\circ}$. DAMPE can measure the energy of protons and nuclei from tens of GeV to about $100 \mathrm{TeV}$. DAMPE measurements are very important for bridging the data measured by space-base experiments and ground-base experiments. In this work, charge measurement method based on Plastic Scintillator detector (PSD) is described. Then analysis progresses on carbon analysis, ultraheavy nuclei and fractional charge particle (FCP) searching based on DAMPE data are presented.

\section{DAMPE Detector}

DAMPE is made up of four sub-detectors as shown in Fig. 1, from the top to the bottom they are: PSD, Silicon-Tungsten Tracker (STK), Bismuth Germanate Oxide Calorimeter (BGO) and a NeUtron Detector (NUD). PSD is designed to measure the charge of cosmic-ray particles and as a veto detector for gamma-ray detection; STK is devoted to reconstruct the trajectories of incident particles; BGO is used to measure the energies of incident particles with a high resolution and to provide electron/hadron identification; NUD is used to separate hadronic shower and electromagnetic shower by measuring neutrons. DAMPE has four trigger modes: unbiased (UB) trigger, minimum ionization particles (MIPs) trigger, high energy (HE) trigger and low energy (LE) trigger. Combining sub-detectors described above, high energy cosmic-rays like electron (positron), gamma-ray and nuclei can be well identified, incident angles and energies of these particles can be well measured. DAMPE has been running smoothly in space more than three and half years, Fig. 2 shows the event number of all events (circles), HE trigger events (squares) and LE trigger events (triangles) per day recorded by DAMPE. The detailed descriptions about DAMPE mission and DAMPE detector calibration can be found in Ref. [3, 4].

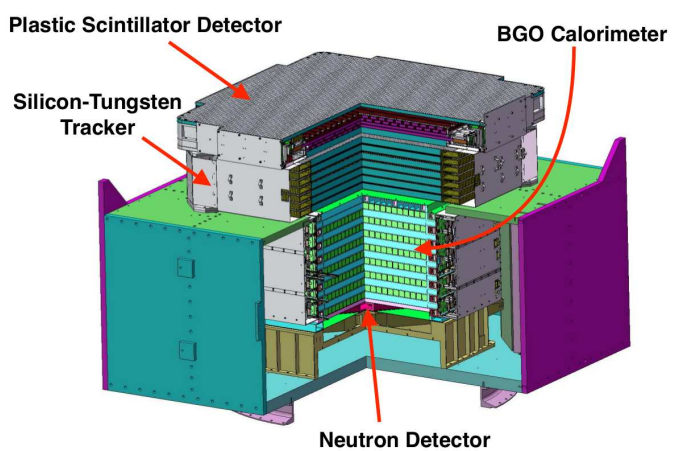

Figure 1: Layout of DAMPE detector.

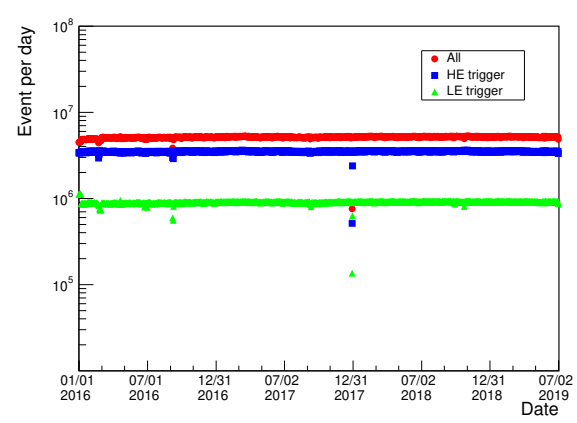

Figure 2: Number of triggered all (circle), highenergy (square), low-energy (triangle) events per day recorded by DAMPE. 


\section{Charge measurement}

PSD is the major sub-detector of DAMPE providing the charge information of incident cosmicray particle. According to Bethe-Bloch formula [11], energy loss of high energy charged particle passing through the matter is proportional to the square of its electric charge. Therefore, the charge of incident particle can be extracted by comparing its energy deposition to MIPs's.

After a series of calibration steps (detailed PSD calibration can be found in Ref. [12]), energies of each PSD scintillator bar and light attenuation functions are obtained. Typical light attenuation behaviors of left (triangles) and right (squares) side of a PSD bar are shown in Fig. 3, where the data points are most probable values (MPV) of Laudau functions used to fit the energy distribution of each hit position slice. Two kinds of functions are used to fit the profiles of Fig. 3, i.e. an exponential function plus a 3rd-order polynomial function (EP3) and a 3rd-order spline function (SP3), which are depicted by the dashed and solid lines, respectively. Both EP3 and SP3 functions can well describe the light attenuation behaviors of PSD bars. The combined energy $\left(E^{c}=\sqrt{E^{L} \times E^{R}}\right)$ of the PSD bar is shown by open circles, and its attenuation function is calculated from attenuation functions of left side and right side, i.e. $A^{C}(x)=\sqrt{A^{L}(x) \times A^{R}(x)}$, where $x$ is the hit position given by a selected track.

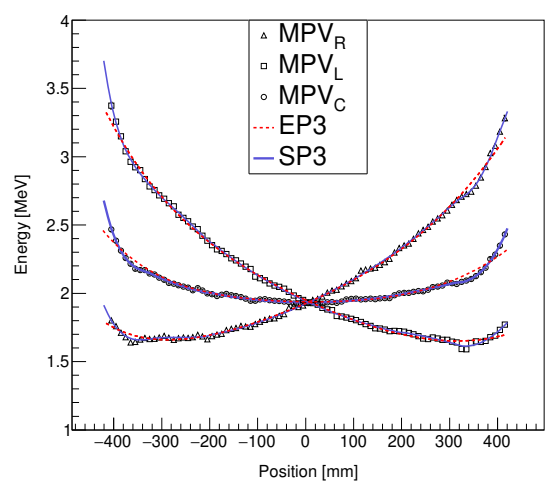

Figure 3: MPVs of Landau functions fitted from the energy distribution of each hit position slice of left (triangles), right (squares) and combined (circles) side of a PSD bar. The fitted EP3 and SP3 functions are shown by dashed and solid lines, respectively.

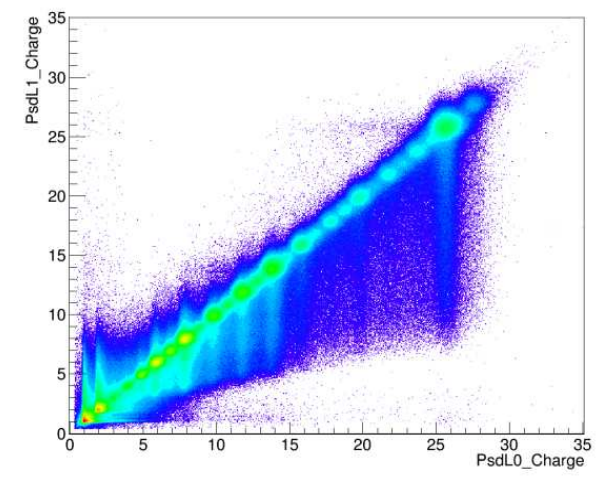

Figure 4: Scatter plot of charges reconstructed by the top layer and the bottom layer of PSD.

The charge $\left(Q_{\text {rec }}^{L / R / C}\right)$ of particle incident on a PSD bar could be extracted by the following expression:

$$
Q_{\text {rec }}^{L / R / C}=\sqrt{\frac{E^{L / R / C}}{A^{L / R / C}(x)} \times \frac{\mathrm{S}}{l}},
$$

where, $E^{L / R / C}$ is the energy of left/right/combined side of a PSD bar, $l$ is the path length of the particle inside the volume of the PSD bar (path length calculation and PSD detector alignment is presented in Ref. [8] of this conference), $\mathrm{S}=10 \mathrm{~mm}$ is the thickness of the PSD bars, $A^{L / R / C}(x)$ is the light attenuation function. Track selection is a key part for obtaining the associated path length and hit position. General track selection method is described in Ref. [10] of this conference. 
The reconstructed charge (Eq. 3.1) still needs quenching effect correction for heavy cosmicray nuclei, then the final charge of incident particles can be obtained. The detailed quenching effect correction method can be found in Ref. [9, 12]. Fig. 4 shows a scatter plot of charges measured by top layer and bottom layer of PSD respectively, in which nuclei from $\mathrm{H}$ to $\mathrm{Ni}$ are well separated. Fig. 5 shows a one-dimensional charge spectrum with combining the charge information of both PSD layers, obtained charge resolutions of cosmic-ray nuclei is comparable to these of AMS02 experiment [9].

Fig. 6 shows the correlation of PSD charge and BGO total energy of flight data. Based on such distribution and Geant 4 based simulation, number of events for different elements, background, acceptance and reconstruction efficiency in different energy ranges can be obtained. In general, the flux $(\Phi)$ of various cosmic-ray elements can be achieved according to Eq. 3.2,

$$
\Phi\left(E_{i}, E_{i}+\Delta E\right)=\frac{N_{i}}{\Delta E_{i} A_{E f f, i} T_{e x p}}
$$

where $\Delta E_{i}$ is the width of $i$-th energy bin, $N_{i}$ is the number of nuclei after background subtraction and unfolding correction, $A_{E f f, i}$ is the effective acceptance, $T_{\exp }$ is the exposure time. For DAMPE cosmic-ray flux analysis, events in South Atlantic Anormaly (SAA) region are excluded. Preliminary proton flux and helium flux results based on DAMPE flight data are presented in Ref. [5] and Ref. [6] of this conference, respectively.

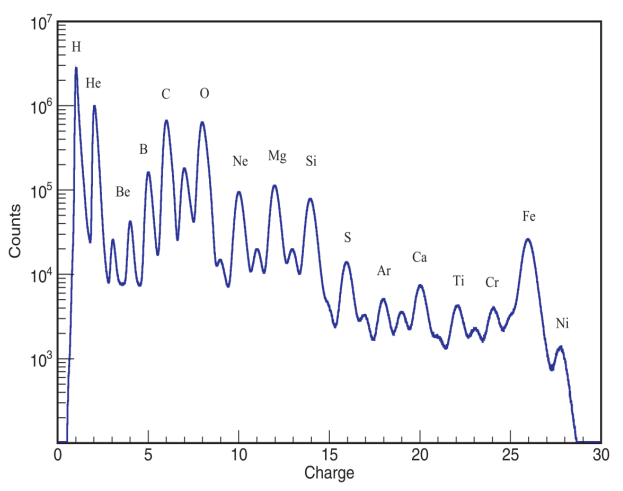

Figure 5: Charge spectrum with combining measurements by both layers of PSD.

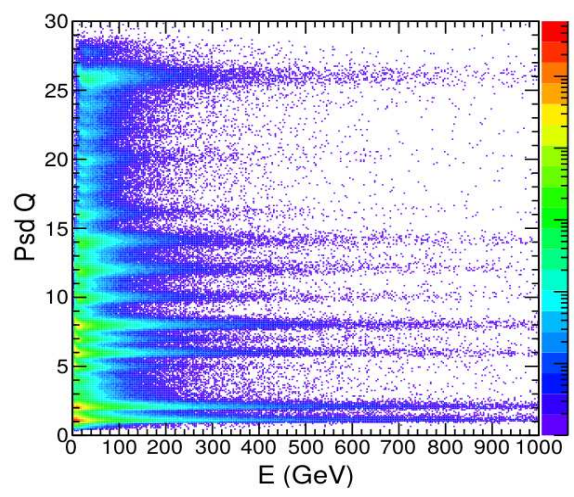

Figure 6: Reconstructed charge versus BGO energy.

\section{Carbon nuclei analysis status}

Boron to Carbon flux ratio is very important for understanding the cosmic ray propagation in the Galactic medium, since Boron is a pure secondary produced by Carbon spallation. HE trigger events are used in the Carbon flux analysis. A global track (BGO track constrained by STK track) according to following criteria is selected: a) contained in the fiducial volumes of PSD and BGO; b) PSD strip hit by the track should be the one with largest energy among neighboring strips; c) charge difference of both PSD layers is required to be less than 2. In order to select events with proper hadronic shower development in BGO, the BGO layer with maximum layer energy (layer energy means adding energies of $22 \mathrm{BGO}$ bars of same layer together) is required within first nine 
BGO layers. The bar with maximum energy in top nine layers should not be in last or first two BGO columns.

Fig. 8 shows the effective acceptance for Carbon nuclei with all selection cuts as a function of primary energy. The effective acceptance is about $0.09 \mathrm{~m}^{2} \mathrm{sr}$ above $300 \mathrm{GeV}$. In order to verify the consistency between MC data and flight data, total BGO energy, energy in each BGO layer, shower profile and its RMS, trigger efficiency and tracking efficiency are compared between flight data and MC data. Some refinements of the MC simulation are necessary in order to get full data-MC agreement. The more detailed analysis can be found in Ref. [13] of this conference.

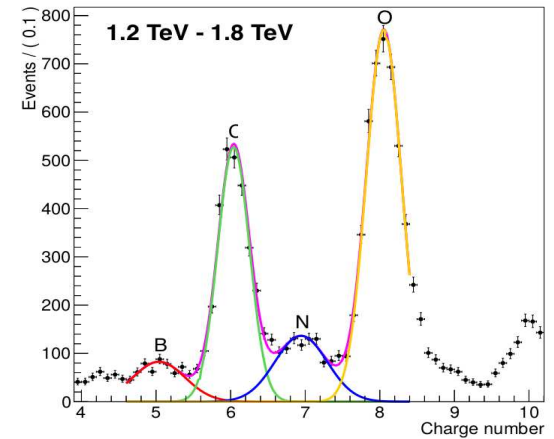

Figure 7: Charge spectra for $\mathrm{Z}=5-8$ nuclei in energy range of 1.2-1.8 TeV.

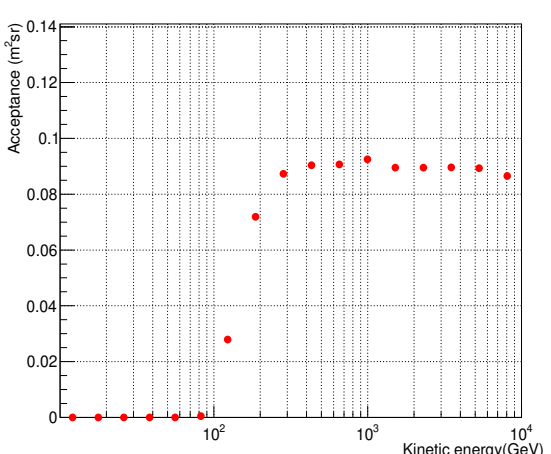

Figure 8: Effective acceptance of carbon nuclei as a function of primary energy.

\section{Ultra-heavy cosmic-ray (UHCR)}

Thanks for larger dynamic-range design of PSD, the energy depositions of cosmic-ray nuclei heavier than Ni can also be covered by PSD. In this analysis, only HE trigger events are used. A global track is selected requiring the following conditions: a) Four STK hits in both XOZ and YOZ plane at least; b) STK energy (sum of energies on the track hits) is the largest one among tracks; c) charge difference of both PSD layers is required to be less than 1. After the track selection, the charge in both top layer and bottom layer of PSD can be obtained, respectively. Fig. 9 shows the preliminary charge spectrum of UHCR using 36 months of flight data, in which the charge peaks of $\mathrm{Ge}(\mathrm{Z}=32), \mathrm{Se}(\mathrm{Z}=34), \mathrm{Sr}(\mathrm{Z}=38)$ and $\mathrm{Zr}(\mathrm{Z}=40)$ are visible clearly. Nevertheless, the statistics of UHCR are very limited. Further studies in order to improve UHCR efficiency and charge reconstruction for nuclei beyond $\mathrm{Zr}$ are on going.

\section{Fractional charge particle (FCP) searching}

According to the standard model, quarks fundamental constituent of matter have either $1 / 3$ or $2 / 3$ of the unit charge, and the quarks are not allowed to appear in isolation due to so-called color confinement. Any observation of free FCP would mean new physics beyond standard model.

MIPs trigger events are used in this analysis. Following selection criteria are applied in order to exclude the background events as much as possible: a) STK track in fiducial volume of PSD and 


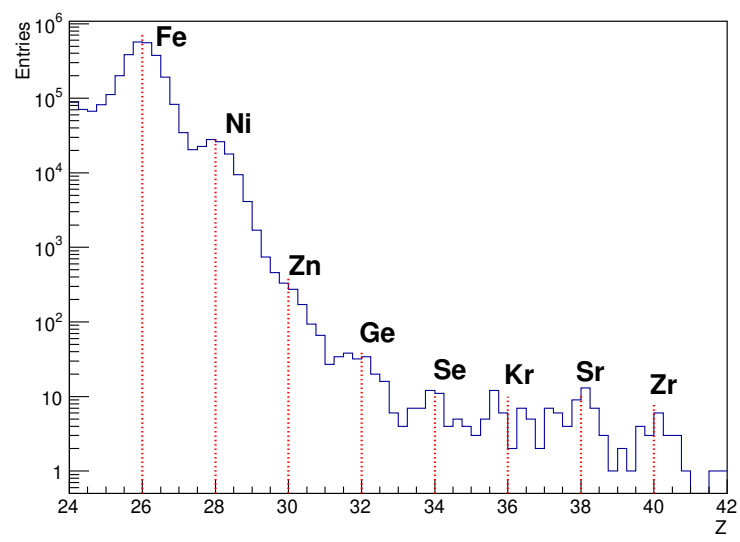

Figure 9: Preliminary charge spectrum of ultra-heavy cosmic-ray nuclei. these events are needed.
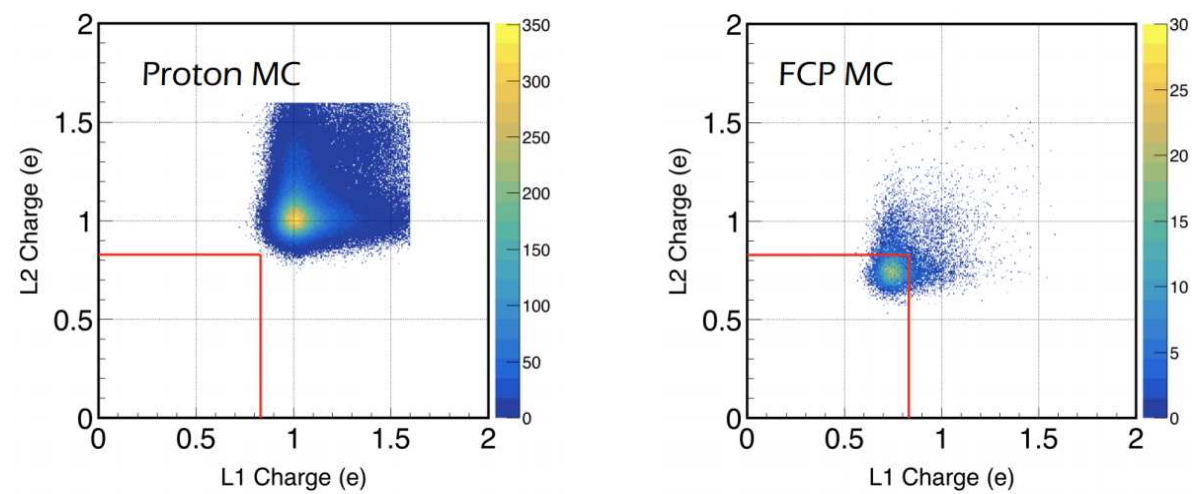

Figure 10: 2D charge correlation for MC proton (left panel) and MC FCP (right panel).

BGO; b) with smallest $\chi^{2} / \mathrm{NDF}$; c) excluding events with charge larger than 1.6 in any PSD layer; d) excluding events with BGO total energy larger than 40 MIPs; f) each BGO layer has one hit (one hit means one BGO bar has valid signals) at least and total BGO hit number is required to be less than 30. After all these cuts, the charges are reconstructed by both PSD layers. Fig. 10 shows charge correlation of both PSD layers of MC proton data (left panel) and MC FCP (assuming FCP with $2 / 3$ of the unit charge) (right panel). In each panel, the red square defines the possible signal region $(Z<0.83)$ for FCP. For MC proton data sample, there is no event in signal region after applying the abovementioned selection cuts. For MC FCP data, most of events are in the signal region. In flight data, we found a few events in the FCP signal region with same analysis procedure. The charge reconstructed for these events can be the effect of mis-matching between track and PSD geometry (mainly on the PSD corner) or signal distortion near the SAA region. More studies about 
allows the measurement of electric charge of cosmic-ray nuclei from $\mathrm{H}$ to $\mathrm{Ni}(\mathrm{Z}=28)$. Carbon nuclei candidates have been selected in the available data sample in different BGO energy ranges. MC validation, studies on background and flux analysis are going on. The ultra-heavy nuclei analysis based on PSD signal has began. Exploiting the PSD features the charge measurement up $\mathrm{Zr}(\mathrm{Z}=40)$ is possible. Cosmic-ray with fractional charge have been studied in DAMPE MC data and flight data. Some events were found in FCP signal region, more detailed investigations for each such event are needed.

\section{References}

[1] Drury L., Astropart. Phys. 39-40 (2012) 52.

[2] Grenier I., et al., Ann. Rev. Astron. Astrophys. 53 (2015) 199.

[3] Chang J., et al, Astropart. Phys. 95 (2017), 6.

[4] G. Ambrosi, et al., Astroparticle Physics 106 (2019) 18.

[5] Yue C., et al., PoS(ICRC2019)163.

[6] M. Di Santo, et al., PoS(ICRC2019)058.

[7] Ma P.X., et al., Research in Astronomy and Astrophysics, 19 (2019) 82.

[8] Ma P.X., et al., PoS(ICRC2019)100.

[9] Dong T.K., et al. Astroparticle Physics 105 (2019), 31-36.

[10] Dong T.K., et al., PoS(ICRC2019)063.

[11] Patrignani C., et al. (Particle Data Group), Chin. Phys. C, 40 (2016), 100001.

[12] Ding M., et al., Research in Astronomy and Astrophysics, 19 (2019), 47.

[13] Wu L.B., PoS(ICRC2019)156. 